\title{
Baboon syndrome caused by anti-haemorrhoidal ointment
}

\author{
Eugénia Matos-Pires ${ }^{1} \mathbb{D}$, David Pina-Trincão ${ }^{2}$, Susana Brás ${ }^{1}$ and Lurdes Lobo ${ }^{1}$ \\ ${ }^{1}$ Department of Dermatology and Venereology, Hospital de Santo António dos Capuchos, Centro Hospitalar de Lisboa Central, 1169-050, Lisbon, Portugal \\ and ${ }^{2}$ Department of Immunoallergology, Hospital de D. Estefânia, Centro Hospitalar de Lisboa Central, 1169-050, Lisbon, Portugal
}

doi:10.1111/cod.12880

Key words: amethocaine; anogenital; baboon syndrome; caine mix; case report; cinchocaine; dibucaine; tetracaine; local anaesthetics; SDRIFE; systemic allergic dermatitis.

Systemic allergic dermatitis caused by local anaesthetics is quite uncommon, and has been mainly attributed to cinchocaine (dibucaine) (1, 2). We report an unusual case of baboon syndrome induced by an anti-haemorrhoidal ointment, most likely attributable to tetracaine (amethocaine) in a patient previously sensitized both to amide and ester local anaesthetics by skin contact.

\section{Case Report}

A 56-year-old man developed widespread dermatitis on the buttocks, skin folds and trunk 2 days after application of Hemofissural $^{\circledR}$ (tetracaine 1\%) ointment on the perianal area for the treatment of haemorrhoids. Two weeks before, the patient had applied Faktu ${ }^{\circledR}$ (cinchocaine 1\%) for the same purpose. He had used several anti-haemorrhoidal ointments for many years, without any severe complications, and with only occasional perianal itch. Physical examination showed an indurated, brightly erythematous and well-demarked plaque with small vesicles and pustules on the buttocks and perianal skin, a diffuse symmetrical erythematous papular eruption on large body folds, and erythematous exudative plaques with vesicles on the trunk. The patient complained of pruritus, with no other symptoms. The dermatitis resolved completely in 8 days with topical and systemic steroids and withdrawal of the anti-haemorrhoidal ointments.

Patch tests with the Portuguese baseline series [includes caine mix III $10 \%$ pet. (benzocaine,

Correspondence to: Dr Eugénia Matos-Pires, Department of Dermatology and Venereology, Hospital de Santo António dos Capuchos, Alameda Santo António dos Capuchos, 1169-050 Lisboa, Portugal. Tel: +351213136497. Email: eugeniampp@gmail.com

Conflict of interest: The authors declare no conflicts of interests.
Table 1. Local anaesthetic series and ointments brought in by the patients, and patch test reactions on day 4

\begin{tabular}{ll}
\hline Local anaesthetic series & Reaction \\
\hline Benzocaine $5 \%$ (pet.) & Negative \\
Tetracaine $\mathrm{HCl} 1 \%$ (pet.) & +++ \\
Cinchocaine $\mathrm{HCl} 5 \%$ (pet.) & +++ \\
Lidocaine $5 \%$ (pet.) & Negative \\
Procaine $\mathrm{HCl} 1 \%$ (pet.) & Negative \\
Caine mix II 3\% (pet.) (cinchocaine, lidocaine, and & +++ \\
$\quad$ tetracaine) & Negative \\
Caine mix I $2 \%$ (pet.) (procaine and lidocaine) & Negative \\
Prilocaine $\mathrm{HCl} 5 \%$ (pet.) & Negative \\
Lidocaine $\mathrm{HCl} 5 \%$ (pet.) & +++ \\
Caine mix III 10\% (pet.) (benzocaine, cinchocaine, & \\
$\quad$ and tetracaine)* & +++ \\
Faktu $^{\circledR}$ 'as is' & +++ \\
Hemofissural & 'as is' \\
\hline
\end{tabular}

$\mathrm{HCl}$, hydrochloride.

* Included in baseline series.

cinchocaine, and tetracaine); Chemotechnique Diagnostics, Vellinge, Sweden], a local anaesthetic series (BIAL Aristegui, Trofa, Portugal) (Table 1) and Faktu ${ }^{\circledR}$ and Hemofissural ${ }^{\circledR}$ ointments 'as is' were applied with Finn Chambers ${ }^{\circledR}$ on Scanpor ${ }^{\circledR}$ tape on the upper back. After $48 \mathrm{~h}$ of occlusion, readings were performed on day (D) 2 and D4 (no later readings were performed). Patch test results are shown in Table 1; allergic contact dermatitis caused by tetracaine and cinchocaine was confirmed.

\section{Discussion}

An individual sensitized to a contact allergen may react in several ways after systemic exposure to the same allergen or a cross-reacting molecule (1). Generalized eruptions, with diffuse erythema of the buttocks and large body folds, namely, baboon syndrome (caused by contact allergens, with previous skin sensitization) or 
symmetrical drug-related intertriginous and flexural exanthema (SDRIFE) (caused by drugs, possibly even at the first exposure), are rare but well-recognized variants of systemic allergic dermatitis, mainly caused by metals and systemic drugs, mostly antibiotics (1). Few cases have been associated with local anaesthetics (mainly cinchocaine) (1). Direct application on the rectal mucosa and the occlusion offered by the intergluteal fold favour systemic drug absorption $(3,4)$.

According to their chemical structure, local anaesthetics can be classified into two major groups, namely, esters (e.g. tetracaine) and amides (e.g. lidocaine and cinchocaine), although cinchocaine belongs to a particular subgroup of amides (2). The different structures make cross-reactivity between esters and amides unlikely. However, concomitant sensitization to both groups has been reported $(2,5)$. In our patient, simultaneous reactivity to cinchocaine and tetracaine may be attributable to concomitant sensitization, because of the use of multiple perianal ointments during the previous years (2). According to the patch test results only, we cannot establish which drug was responsible for the baboon syndrome. Nevertheless, considering the time between the development of the dermatitis and the application of Hemofissural $^{\circledR}$, we believe that the baboon syndrome was caused by tetracaine. To our knowledge, this would be the second case attributable to this topical anaesthetic (6).

In conclusion, this case shows that the perianal skin fold might facilitate cutaneous sensitization to (ingredients of) anti-haemorrhoidal ointments, that subsequent mucosal absorption is an important route for inducing systemic contact dermatitis, potentially presenting as baboon syndrome or SDRIFE, that cutaneous sensitization to different local anaesthetics is likely in patients with longstanding perianal disease, and that patch testing with only benzocaine as a screener in the baseline series is insufficient to diagnose these cases. Therefore, including a caine mix in the baseline series may be beneficial.

\section{References}

1 Häusermann P, Harr T H, Bircher A J. Baboon syndrome resulting from systemic drugs: is there strife between SDRIFE and allergic contact dermatitis syndrome? Contact Dermatitis 2004: 51: 297-310.

2 Brinca A, Cabral R, Gonçalo M. Contact allergy to local anaesthetics - value of patch testing with a caine mix in the baseline series. Contact Dermatitis 2013: 68: $156-162$.

3 Marques C, Faria E, Machado A et al. Allergic contact dermatitis and systemic contact dermatitis from cinchocaine. Contact Dermatitis 1995: 33: 443.

4 Erdmann S M, Sachs B, Merk H F. Systemic contact dermatitis from cinchocaine. Contact Dermatitis 2001: 44: 260-261.
5 Gunson T H, Greig D E. Allergic contact dermatitis to all three classes of local anaesthetic. Contact Dermatitis 2008: 59: $126-127$

6 Huerta Brogeras M, Avilés J A, González-Carrascosa M et al. Tetracaine-induced systemic contact-type dermatitis. Allergol Immunopathol 2005: 33 : 112-114. 